\title{
Electrical and magnetic properties of polyvinyl alcohol-cobalt ferrite nanocomposite films
}

\author{
DHIRAJ KUMAR RANA ${ }^{1}$, SURESH KUMAR SINGH ${ }^{1}$, SHOVAN KUMAR KUNDU ${ }^{1}$, \\ RAM JANAY CHOUDHARY ${ }^{2}$ and SOUMEN BASU ${ }^{1, *}$ \\ ${ }^{1}$ Department of Physics, National Institute Technology, Durgapur 713209, India \\ ${ }^{2}$ Department of Atomic Energy Consortium for Scientific Research, University Grants Commission, Indore 452001, India \\ *Author for correspondence (sou_menbasu@yahoo.com)
}

MS received 29 August 2017; accepted 8 November 2017; published online 4 July 2018

\begin{abstract}
Polyvinyl alcohol (PVA)-cobalt ferrite (CFO) nanocomposite films were synthesized by wet chemical method. The synthesized CFO nanomaterial was characterized by X-ray diffraction (XRD) and transmission electron microscopy (TEM), which confirm the formation of pure phase nanoparticles with cuboid shape. The variation in AC conductivity is measured as a function of frequency (within the range of $100 \mathrm{~Hz}-1 \mathrm{MHz}$ ) and temperature (from 303 to $403 \mathrm{~K}$ ). It was observed that the frequency exponent, $S$, decreases with increasing temperature, which is explained by correlated barrier hopping $(\mathrm{CBH})$ conduction mechanism. Frequency dependence of real and imaginary parts of the dielectric permittivity of PVA-CFO nanocomposite films for different temperatures is studied on the basis of the modified Cole-Cole model. The dielectric permittivity of the samples reveals an increasing tendency with the concentration of CFO filler in PVA matrix. A high value of $89 \mathrm{emu} \mathrm{g}^{-1}$ saturation magnetization and 652 Oe coercivity are observed in CFO nanoparticle. The coercivity increased in PVA-CFO nanocomposite when compared to CFO nanoparticle, which is expected due to inverse magnetostrictive effect. The increasing tendency of dielectric constant and magnetization of the nanocomposites with the increasing CFO concentration enhances the potential of applications in miniaturization of the antenna system and electromagnetic shielding materials.
\end{abstract}

Keywords. Nanocomposite; nanoparticles; PVA; CFO; CBH.

\section{Introduction}

Development of novel materials with some new properties is one of the main targets of advanced materials research. Synthesis of different nanomaterials and study of their remarkable properties is of much interest due to their enormous practical applications of various fields like engineering, technology, medical, etc. An appropriate method to develop composite materials is to combine two and more individual systems properties in a single material [1-3]. This combination of desired physical and chemical inherent features creates multifunctional nanocomposite with extraordinary properties $[4,5]$. The research on nanocomposite materials is increasing dayby-day because of their fascinating applications for different fields like electronic devices, drug delivery, sensors, electromagnetic shielding, etc. [6].

Among the different types of magnetic materials, cobalt ferrite (CFO) with inverse spinel structure having a chemical formula $\mathrm{CoFe}_{2} \mathrm{O}_{4}$ is one of the emerging and promising magnetic materials in terms of high coercive field, high saturation magnetization value, excellent chemical stability, mechanical hardness and large magnetocrystalline anisotropy. It is used in magnetic recording media, in medical applications including magnetic resonance imaging (MRI), targeted drug delivery, magnetic separation, biosensors and hyperthermia $[7,8]$. Polymers have many interesting properties, such as easily transforming into any shape, light weight, corrosion resistance, environment-friendly, high mechanical strength and much more [9]. The advantage of polymer-based magnetic material nanocomposites is polymer matrix trapped the magnetic nanoparticles and restrict the agglomeration of magnetic nanoparticles, which improve the chemical stability [7]. Polyvinyl alcohol (PVA) is a semi-crystalline, non-toxic, biocompatible, biodegradable, water-soluble polymer with an excellent chemical stability [10].

The embedding of CFO nanoparticles with PVA matrix due to their significant potential applications were investigated earlier and the effects of the polymer matrix and the synthesis methods of shape, size and magnetic properties of cobalt ferrite particles were studied [11-13]. Sinha et al [14] have reported the electrical transport properties of PVAselenium nanocomposite films at and above room temperature and reveal the potentiality of PVA nanocomposites for electromagnetic shielding application.

In this work, we have synthesized the PVA-CFO nanocomposite films with different $\mathrm{CFO}$ concentrations by wet chemical method. The effects of CFO on the electrical and magnetic properties of PVA-CFO nanocomposites are 
investigated in detail. The results would be helpful for further studies on physical and biocompatibility properties of these nanocomposites to apply in miniaturization of the patch antenna, electromagnetic shielding and biomedical applications.

\section{Experimental}

\subsection{Synthesis of CFO nanoparticles}

CFO nanoparticles are synthesized by wet chemical method using raw materials of cobalt(II) chloride hexahydrate $(10 \mathrm{mM})$ and iron(III) chloride anhydrous $(20 \mathrm{mM})$ in 1:2 molar ratio were mixed in $20 \mathrm{ml}$ double-distilled deionized water. A solution of sodium hydroxide $(150 \mathrm{mM})$ in $100 \mathrm{ml}$ deionized water was prepared and added slowly to the salt solution. During the reaction, the $\mathrm{pH}$ of the solution was constantly monitored. The reactant was constantly stirred using the magnetic stirrer until the $\mathrm{pH}$ level reached to 12. A specified amount of acetic acid was added to the solution as a capping agent. The resultant colloidal solution was then heated to a reaction temperature of $80^{\circ} \mathrm{C}$ and continuously stirred for $1 \mathrm{~h}$. The product was then cooled to room temperature. To remove sodium and chlorine compounds, the solution was centrifuged at $6000 \mathrm{rpm}$ for $15 \mathrm{~min}$. The precipitates were washed with deionized water and ethanol for several times. Then, the black precipitates were dried at $100^{\circ} \mathrm{C}$. Afterwards, the acquired substance was ground into a fine powder and calcined at $600^{\circ} \mathrm{C}$ for $10 \mathrm{~h}$ and finally, cobalt ferrite nanoparticles were formed.

\subsection{Synthesis of PVA-CFO nanocomposite films}

PVA was directly purchased from Sigma-Aldrich. For the preparation of $10 \mathrm{wt} \%$ PVA solutions, the calculated amount of PVA powder was added in deionized water and stirred at temperature $60^{\circ} \mathrm{C}$ for completely dissolving the PVA powder in deionized water. In this process, little amount of ethanol was added to the solution to increase the solubility rate of PVA. For the preparation of PVA-CFO nanocomposite films, varying $\mathrm{CFO}$ concentrations (4, 8 and $10 \mathrm{wt} \%$ ) were added in PVA solution and tip sonicated till the better dispersion of CFO nanoparticles in PVA solution. The solution was poured on a glass slide by drop-casting and dried at room temperature for overnight. Finally, the PVA-CFO nanocomposite films were formed. Here, the sample's name is given as $\mathrm{S}-0$ for pure PVA film, and S-4, S-8 and S-10 for PVA-CFO (4, 8 and $10 \mathrm{wt} \%$ ) nanocomposite films, respectively. The thickness of PVA-CFO nanocomposite films is measured by digital screw gauge and the thicknesses of the nanocomposites are $25.35,32.69,27.25$ and $43.91 \mu \mathrm{m}$ for $\mathrm{S}-0, \mathrm{~S}-4, \mathrm{~S}-8$ and $\mathrm{S}-10$, respectively.

\subsection{Characterization techniques}

The phase identification of the CFO nanoparticles was studied by X-ray diffraction (XRD) pattern analysis. The XRD pattern was recorded by $\mathrm{X}^{\prime}$ Pert Pro X-ray diffractometer PANLYTICAL, Almelo, The Netherlands, using nickel-filtered $\mathrm{CuK} \alpha$ radiation $(\lambda=1.5406 \AA)$ in the $2 \theta$ range from 20 to $80^{\circ}$. The transmission electron microscopy (TEM) analysis was performed by HRTEM, JEOL 2011, to estimate particle size, morphology and the crystallinity of the prepared CFO nanoparticles. For the TEM study, the CFO powder was ground by an agate mortar, dispersed in ethanol by the ultrasonic bath and cast one drop on a carbon-coated copper grid. The AC electrical properties of PVA-CFO nanocomposites were recorded using an Agilent E4980A LCR meter with frequency range of $100 \mathrm{~Hz}-1 \mathrm{MHz}$ and temperature from 303 to $403 \mathrm{~K}$. The magnetic hysteresis loops (M-H curve) of the
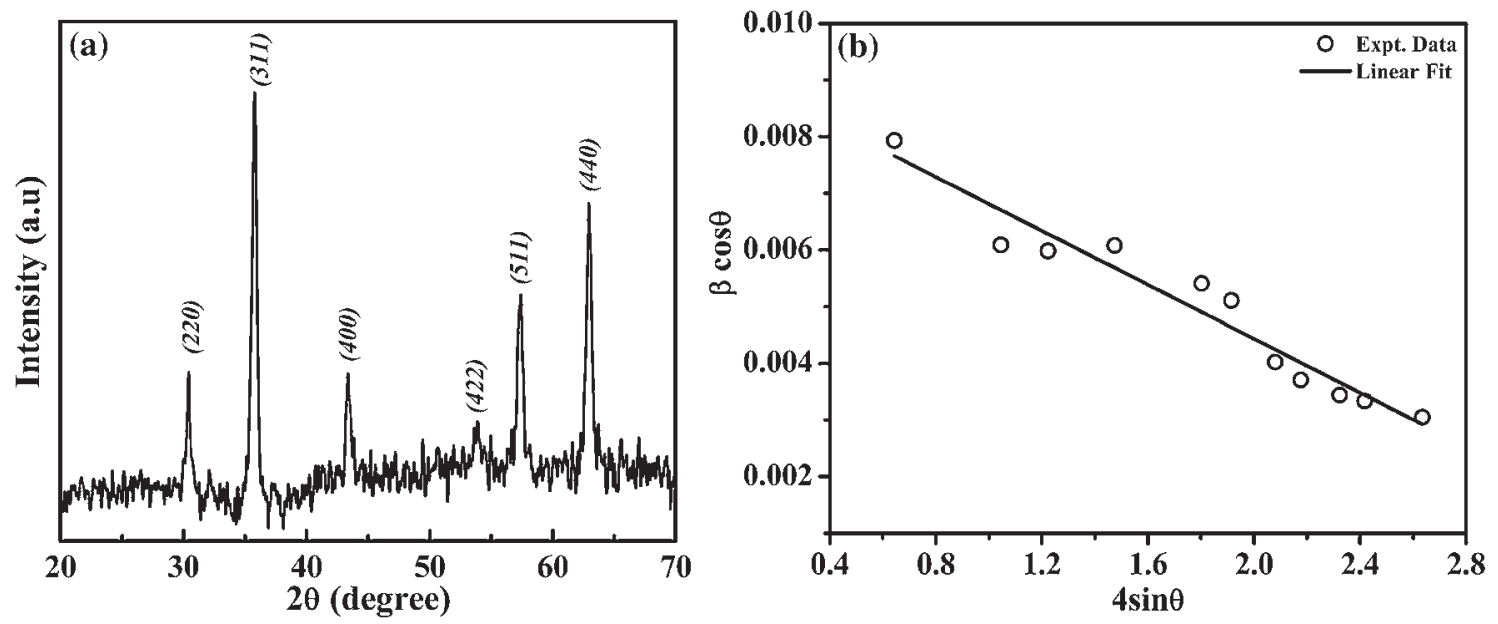

Figure 1. (a) X-ray diffraction (XRD) pattern of CFO nanoparticles. (b) Williamson-Hall plot of XRD pattern of CFO nanoparticles. 
samples were measured by Quantum Design SQUID VSM 7 Tesla Magnetometer.

\section{Result and discussion}

The XRD pattern of CFO nanoparticles is shown in figure 1a. All the lines were identified with those of pure cobalt ferrite nanoparticles as in JCPDS card no. 22-1086. All the $(h k l)$ planes of CFO nanoparticles are shown against the intensity peaks of figure 1a. From the XRD pattern analysis, it is observed that the prepared CFO nanoparticles have inverse spinel of face-centered-cubic structure of Fd-3 m space group and space group number 227 . The average crystallite size and lattice strain are estimated by using Williamson-Hall plot, which is shown in figure 1b. Using Williamson-Hall formula as shown in equation (1) and from the graph of $4 \sin \theta v s$. $\beta$ $\cos \theta$, average crystallite size and lattice strain were estimated from the intercept and slope of the straight line fitting of figure $1 \mathrm{~b}$, respectively. Hence, the calculated average crystallite size is $15 \mathrm{~nm}$ and the lattice strain of CFO nanoparticles is -0.0024 .

$$
\beta \cos \theta=\frac{K \lambda}{L}+4 \eta \sin \theta,
$$

where $\beta$ is full-width at half-maxima (FWHM) of the diffraction peaks, $L$ the average crystallite size, $K$ a constant, which is $0.9, \lambda$ the wavelength of X-ray source $(1.5406 \AA$ ) and $\eta$ the lattice strain. The average crystallite size was also estimated from Debye-Scherrer's formula [15] by considering the highest intense (311) diffraction peak only at $17.18 \mathrm{~nm}$.
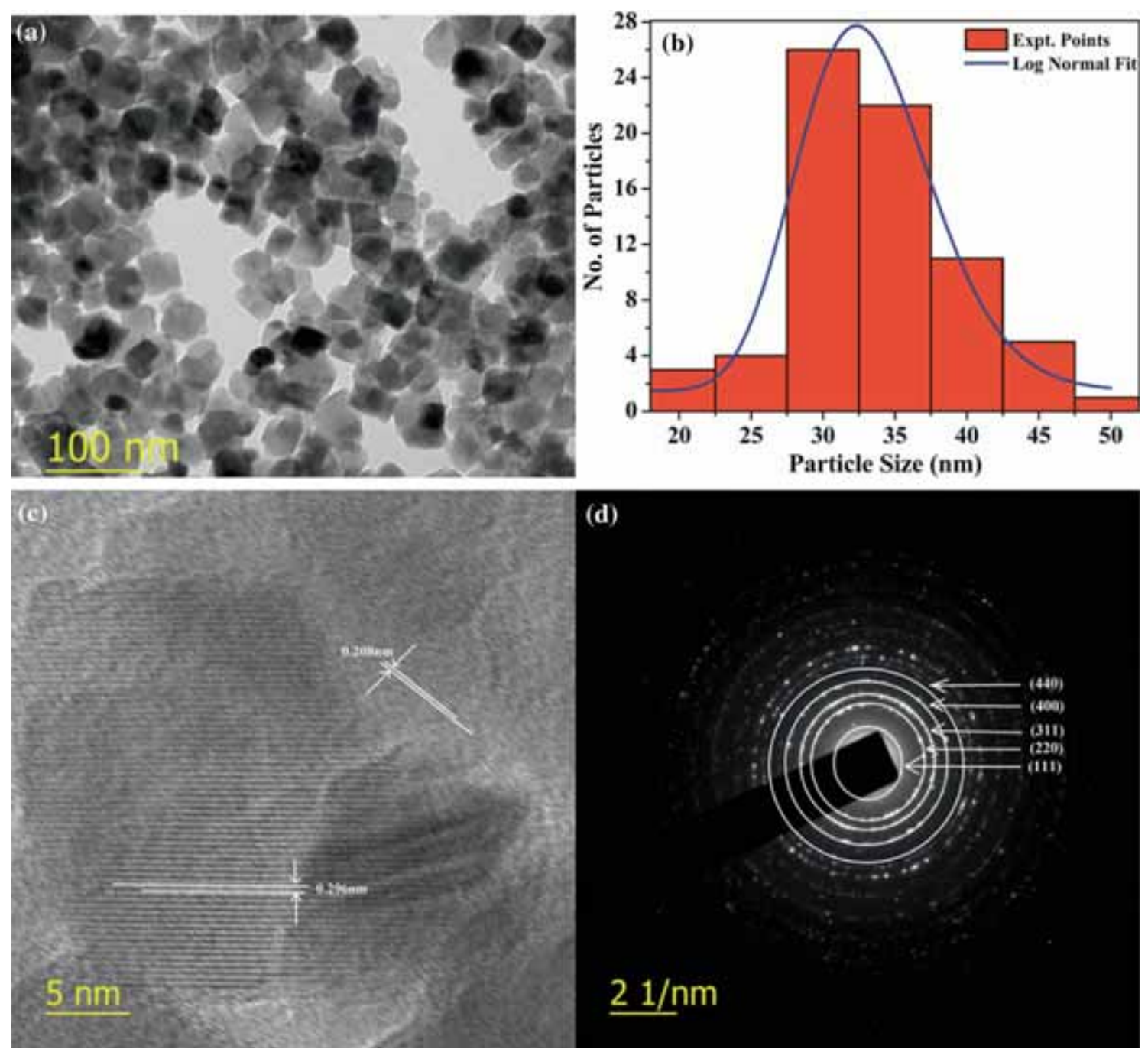

(d)

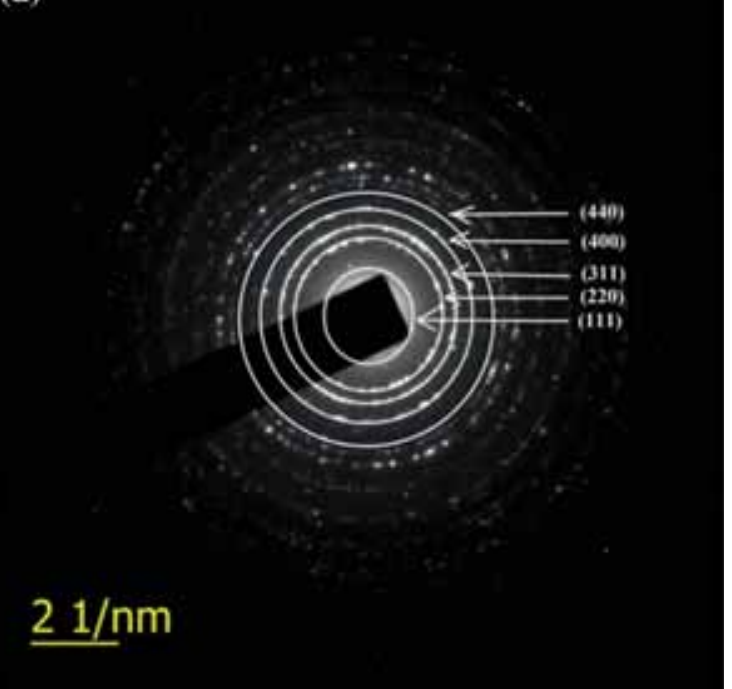

Figure 2. (a) Particle distribution pattern of CFO nanoparticles. (b) Log normal histogram of particles distribution pattern of CFO nanoparticles. (c) High-resolution transmission electron micrograph (HRTEM) of CFO nanoparticles. (d) Selected area electron diffraction pattern of CFO nanoparticles. 
This is consistent with the value estimated from WilliamsonHall plot. The negative value of micro strain suggests that the compressive strain in the crystal lattice may be due to the shape anisotropy of the CFO nanoparticles.

The TEM of the CFO nanoparticles is shown in figure $2 \mathrm{a}$. From the particle distribution pattern of CFO nanoparticles in figure $2 \mathrm{a}$, it is clearly observed that the $\mathrm{CFO}$ nanoparticles are distributed uniformly. The shape of the nanoparticles is not exactly spherical, but it looks like cuboids shape. From standard log normal distribution plot represented in figure $2 \mathrm{~b}$, the average particle size was estimated as $33 \mathrm{~nm}$. Here it is noted that with TEM we measure the particle size, whereas with $\mathrm{XRD}$ one can measure the crystallite size, which is the coherent with diffracting crystalline domains, which is different from particle size. One particle can be constituted by several crystalline domains; this is why always particle size has to be bigger than crystallite (domain) size. Figure $2 \mathrm{c}$ represents the high-resolution transmission electron micrograph (HRTEM) taken from a portion of figure $2 \mathrm{a}$. The lattice spacings of 0.296 and $0.208 \mathrm{~nm}$ are calculated from figure $2 \mathrm{c}$, which represent the (220) and (400) planes of CFO nanoparticles, respectively, and confirm the crystalline nature of the prepared sample.
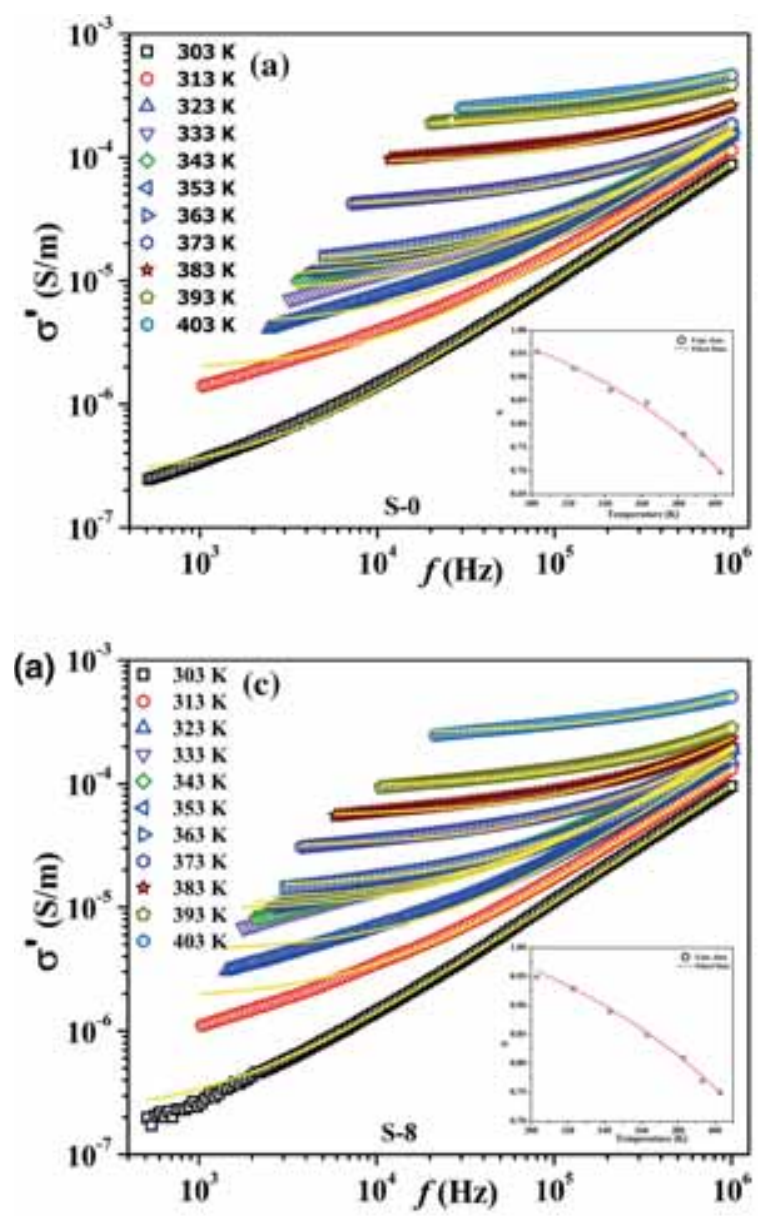

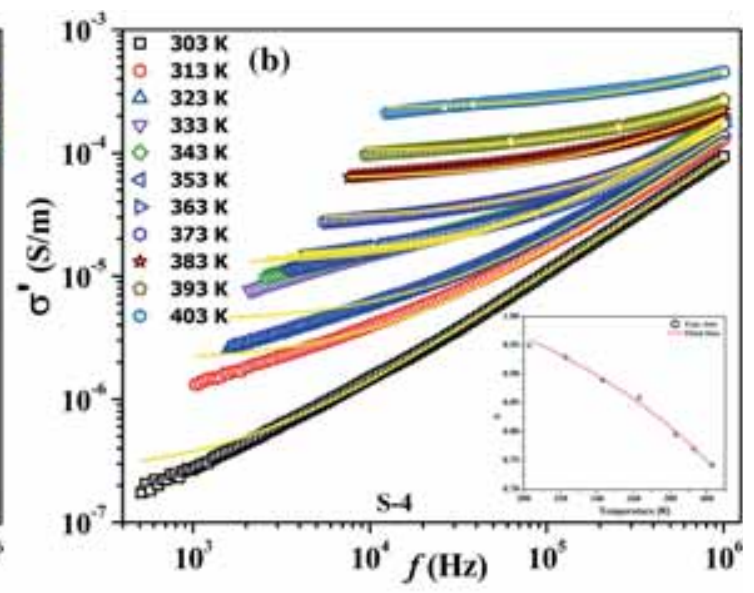

The selected area electron diffraction (SAED) pattern of CFO nanoparticles is shown in figure $2 \mathrm{~d}$, and large number of concentric rings is observed due to the random orientation of crystallites and also confirms the well-crystalline nature of CFO nanomaterials. The concentric rings represent the different $(h k l)$ planes indicated with arrowheads in figure $2 \mathrm{~d}$.

The variation in total measured conductivity $\left(\sigma^{\prime}\right)$ of the PVA-CFO nanocomposite films with frequency range of $100 \mathrm{~Hz}-1 \mathrm{MHz}$ is studied for different temperatures varying from 303 to $403 \mathrm{~K}$. At lower frequency, the conductivity is almost frequency-independent, but becomes predominant at a higher frequency for all the temperatures. In general, many amorphous semiconductors or disordered systems have DC conductivity contribution $\left(\sigma_{\mathrm{dc}}\right)$ besides the AC conductivity. This may be the reason for the frequency independence of conductivity at lower frequency region. The total conductivity at a particular temperature over a wide range of frequency obey the universal dielectric response (UDR) with frequency, which can be expressed as [16-20]:

$$
\sigma^{\prime}=\sigma_{\mathrm{dc}}+\sigma_{\mathrm{ac}}=\sigma_{\mathrm{dc}}+\sigma_{\mathrm{o}} f^{\mathrm{s}}
$$

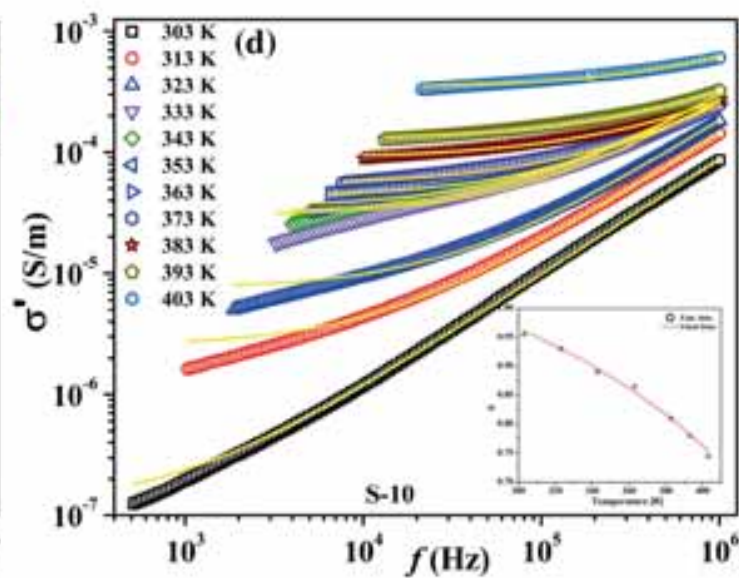

Figure 3. Variation of total measured electrical conductivity with frequency at different temperatures for (a) S-0, (b) S-4, (c) S-8 and (d) S-10. The solid line represents the theoretical fitting. The inset of the figures shows the variation of frequency exponent $S$ (circles) corresponds to temperature with theoretical fitting (solid line). 
Table 1. Parameters estimated from the frequency exponent $(S)$ fitting with temperature at $10 \mathrm{kHz}$ frequency.

\begin{tabular}{lcccc}
\hline Parameter & S-0 & S-4 & S-8 & S-10 \\
\hline$W_{\mathrm{m}}(\mathrm{eV})$ & 0.408 & 0.427 & 0.432 & 0.434 \\
$\tau_{0}(\mathrm{~s})$ & $1.09 \times 10^{-12}$ & $5.38 \times 10^{-12}$ & $7.26 \times 10^{-12}$ & $9.81 \times 10^{-12}$ \\
\hline
\end{tabular}

where $\sigma^{\prime}$ is the total measured conductivity, $\sigma_{\mathrm{dc}}$ and $\sigma_{\mathrm{ac}}$ are the DC and AC contributions of total measured conductivity, respectively, $\sigma_{0}$ a constant, $f$ the frequency and $S$ the frequency exponent, which gives the degree of interaction of charge carriers with the lattice.

Figure $3 \mathrm{a}-\mathrm{d}$ represents the variation in conductivity with frequency of different temperatures for PVA-CFO nanocomposites. The solid line shows the fitting of UDR as in equation (2). From the figure, it is observed that the total conductivity of all the nanocomposites are well-fitted with UDR model and from the fitting, we estimate the frequency exponent $(S)$. At room temperature, the value of $S$ is near unity and with increasing temperature, $S$ is decreasing for all the nanocomposites, which is shown in insets of figure $3 \mathrm{a}-\mathrm{d}$. In general, the nature of conduction process of the disordered system is governed by two physical processes, such as correlated barrier hopping $(\mathrm{CBH})$ [17] and quantum mechanical tunnelling (electron tunnelling [18], small polaron tunnelling [17] and large polaron tunnelling [20]). The variation of $S$ with temperature for different conduction processes is different. Observing the nature of variation in $S$, the exact nature of charge transport mechanism can be confirmed. The frequency exponent, $S$, becomes independent of temperature in electron tunnelling theory. The frequency exponent increases with increasing temperature in small polaron theory. In large polaron theory, the frequency exponent decreases first, then it increases with increasing temperature. According to CBH model, the value of $S$ only decreases gradually with increasing temperature. In the present study, the nature of variation in $S$ suggests that the CBH model is suitable for explaining the experimental data. According to this model, the charge carriers hop between the sites over the potential barrier separating them. The frequency exponent $S$ can be expressed as [17]:

$$
S=1-\frac{6 K_{\beta} T}{\left[W_{\mathrm{m}}-K_{\beta} T \ln \left(\frac{1}{\omega_{\tau_{0}}}\right)\right]},
$$

where $K_{\beta}$ is the Boltzmann constant, $T$ the temperature, $W_{\mathrm{m}}$ the maximum barrier height, $\omega$ the angular frequency and $\tau_{0}$ the relaxation time.

Therefore, the experimental data were studied using equation (3) as a function of temperature keeping $W_{\mathrm{m}}$ and $\omega \tau_{0}$ as parameters. The insets of figure $3 \mathrm{a}-\mathrm{d}$ indicate the experimental data points and solid lines represent the theoretical best fit obtained from equation (3) for different nanocomposites. The values of barrier height $\left(W_{\mathrm{m}}\right)$ and relaxation time $\left(\tau_{0}\right)$ were calculated at a fixed frequency of $10 \mathrm{kHz}$ for all the nanocomposites and are enlisted in table 1. It is observed that the values of both $W_{\mathrm{m}}$ and $\tau_{0}$ are increasing with increase in the $\mathrm{CFO}$ wt $\%$ in PVA matrix.

Frequency dependence of real and imaginary parts of dielectric permittivity with different temperatures of PVACFO nanocomposite films are shown in figures $4 \mathrm{a}-\mathrm{d}$ and $5 \mathrm{a}-\mathrm{d}$, respectively. The high value of real part of dielectric permittivity is observed at low-frequency region. It is also observed that the value of dielectric permittivity increases with increasing temperature and decreases with increasing frequency. It shows the minimum value at higher frequency region. In general, polymer composites are heterogeneous, the heterogeneity suggests the Maxwell-Wagner type of dielectric relaxation [21]. The frequency variation of dielectric permittivity is due to the presence of four types of polarization in different frequency regions. In low-frequency region, interface or space charge polarization takes place. This polarization is basically due to the grain boundaries effect or interface between the electrode and surface of the materials and at high-frequency region, ionic and electronic polarizations will occur [14]. The dielectric dispersion in PVA-CFO nanocomposites are due to the interfacial polarization occurring in electrically inhomogeneous or a heterogeneous system. In this case, interfacial polarization due to Maxwell-Wagner-Sillars effects occurred between the semiconductor nanoparticles filler and the non-conducting polymer matrix. When we apply an electric current to the interface of two dielectric mediums, there is a large difference in conductivities in the interface of semiconductor nanoparticles and non-conducting polymer matrix gives rise to the dipoles and which get polarized and responsible for the dielectric permittivity [22]. The experimental data points of real $\left(\varepsilon^{\prime}\right)$ and imaginary $\left(\varepsilon^{\prime \prime}\right)$ parts of dielectric permittivity with frequency is well-fitted with the modified Cole-Cole equation [23] as expressed in equations (4) and (5) and the fitted curve of real and imaginary parts of dielectric permittivity at five different temperatures is shown in the insets of figures $4 a-d$ and $5 a-d$, respectively. From the least square fitting of modified Cole-Cole equation, the values of $\tau, \beta, m, \sigma_{\mathrm{sp}}$ and $\sigma_{\mathrm{fc}}$ are estimated and summarized in table 2 .

$$
\begin{aligned}
\varepsilon^{\prime}= & \varepsilon_{\infty}+\left[\frac{\left(\varepsilon_{\mathrm{s}}-\varepsilon_{\infty}\right)\left\{1+(\omega \tau)^{\beta} \cos (\beta \pi / 2)\right\}}{1+2(\omega \tau)^{\beta} \cos (\beta \pi / 2)+(\omega \tau)^{2 \beta}}\right] \\
& +\frac{\sigma_{\mathrm{sp}}}{\varepsilon_{0} \omega^{m}},
\end{aligned}
$$



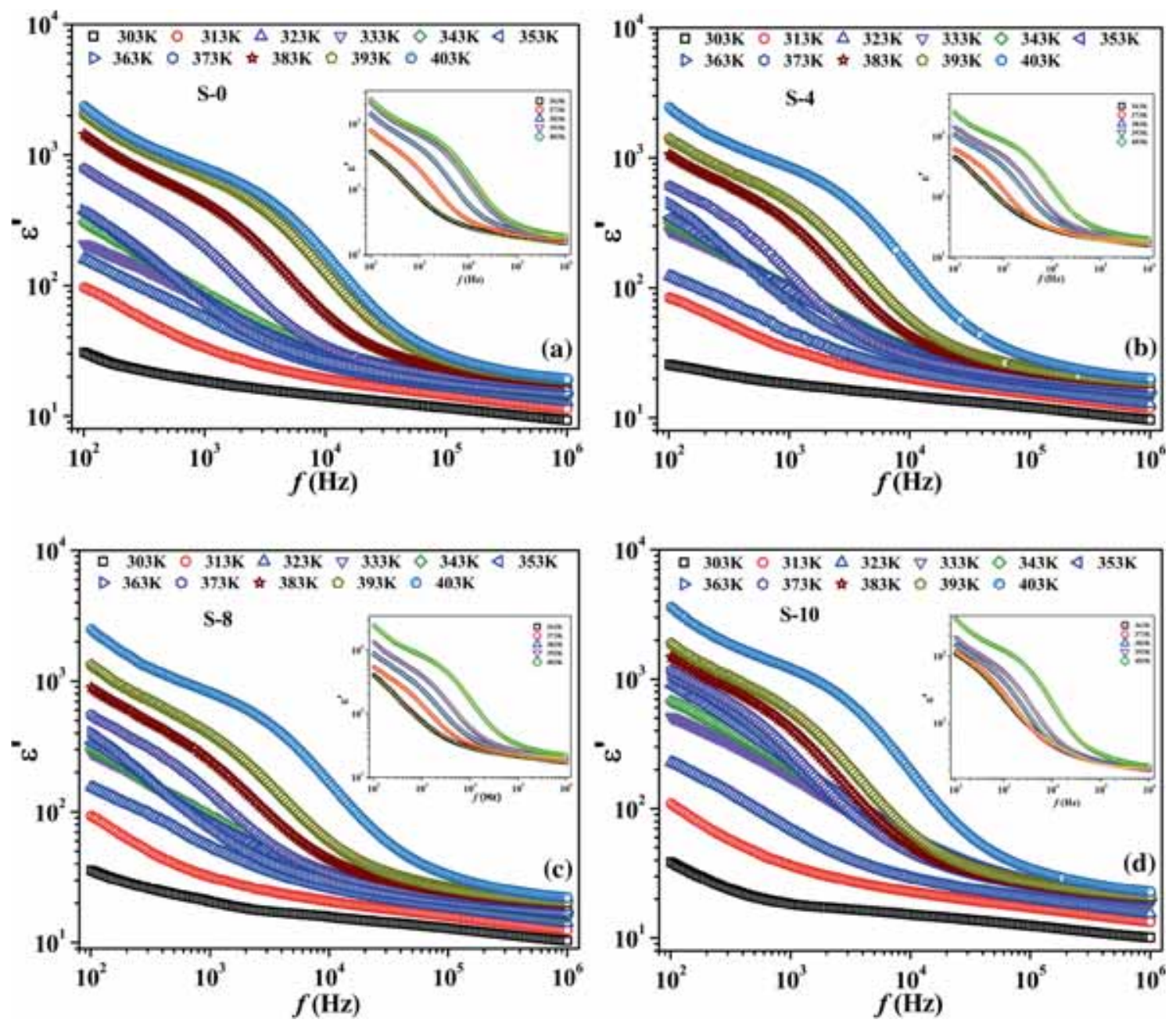

Figure 4. Frequency dependence of real part of dielectric permittivity with different temperatures for (a) S-0, (b) S-4, (c) S-8 and (d) S-10. The inset of the figures shows the fitting of modified Cole-Cole equation with experimental data points.

$$
\begin{aligned}
\varepsilon^{\prime \prime}= & {\left[\frac{\left(\varepsilon_{\mathrm{s}}-\varepsilon_{\infty}\right)(\omega \tau)^{\beta} \sin (\beta \pi / 2)}{1+2(\omega \tau)^{\beta} \cos (\beta \pi / 2)+(\omega \tau)^{2 \beta}}\right] } \\
& +\frac{\sigma_{\mathrm{fc}}}{\varepsilon_{0} \omega^{m}},
\end{aligned}
$$

where $\varepsilon_{\mathrm{s}}$ and $\varepsilon_{\infty}$ are the low- and high-frequency dielectric permittivities, respectively, $\tau$ the relaxation time, $\omega$ the angular frequency, $\beta$ the parameter, which lies between 0 and 1 , $m$ the exponent, $\sigma_{\mathrm{sp}}$ and $\sigma_{\mathrm{fc}}$ are the space and free charge conductivities, respectively.

The variation in real and imaginary parts of dielectric permittivity with temperature at $1 \mathrm{MHz}$ frequency of different PVA-CFO nanocomposites is shown in figure $6 \mathrm{a}$ and $\mathrm{b}$. The nature of variations in dielectric constant is same for pure PVA film (S-0) sample as well as PVA-CFO nanocomposites i.e., S-4 and S-10 samples. A broad hump appeared in S-10 sample at temperature around $350 \mathrm{~K}$ and after that with increasing temperature, dielectric constant increased. This is due to the generation of extra thermal energy, which enhances the mobility of charge carriers [13]. In figure $6 \mathrm{~b}$, a broad peak in the imaginary part of the dielectric permittivity at around $350 \mathrm{~K}$ implied that the nanocomposite shows relaxation behaviour. When the relaxation frequency matches with the applied frequency i.e., $\omega \tau=1$, a peak appeared in the dielectric loss plot called the relaxation peak and it is steeper with increasing temperature. The real and imaginary parts of dielectric permittivity increases with increasing the CFO concentrations in PVA matrix, suggesting control for impedance matching for antenna applications.

The magnetic properties of the nanocomposites are measured by Quantum Design SQUID VSM 7 Tesla system. Magnetic hysteresis was recorded in the magnetic field range of \pm 7 Tesla at 10 and $300 \mathrm{~K}$. Figure 7 depicted the hysteresis behaviour of CFO nanoparticles at 10 and $300 \mathrm{~K}$. The hysteresis loop of CFO nanoparticles at $10 \mathrm{~K}$ shows a complex behaviour of magnetization near zero magnetic fields. The same kind of behaviour of magnetization near zero magnetic fields at low temperature is also observed in 

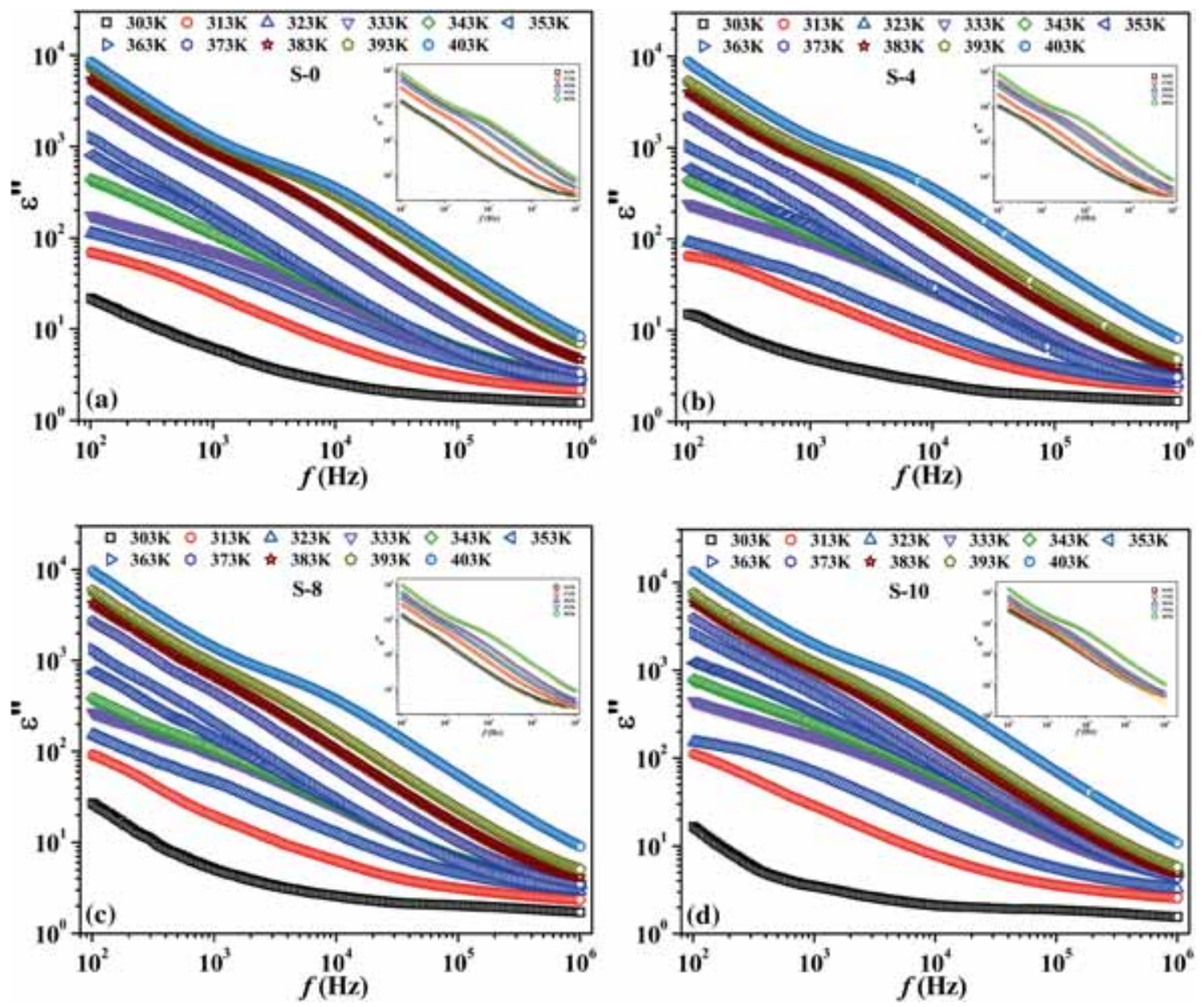

Figure 5. Frequency dependence of imaginary part of dielectric permittivity with different temperatures for (a) S-0, (b) S-4, (c) S-8 and (d) S-10. The inset of the figures shows the fitting of modified Cole-Cole equation with experimental data points.

previous reported work of CFO nanoparticles [24,25]. This type of magnetization behaviour is due to the presence of some soft magnetic phase as well as the hard magnetic phase in the $\mathrm{CFO}$ nanoparticles [24]. At room temperature, the observed saturation magnetization is $89 \mathrm{emu} \mathrm{g}^{-1}$, which is very high when compared to the similar size of CFO nanoparticles [26]. This saturation magnetization value is very close to the bulk value of CFO [27]. We have also estimated the magnetic moment due to one formula unit for CFO nanoparticles from this saturation magnetization value. The magnetic moment of one formula unit for $\mathrm{CFO}$ is found to be $3.74 \mu_{\mathrm{B}}$. Generally, the structure of $\mathrm{CFO}$ is inverse spinel $\left(\mathrm{Fe}^{3+}\left(\mathrm{Co}^{2+} \mathrm{Fe}^{3+}\right) \mathrm{O}_{4}^{2-}\right)$ and for all inverse ferrites, the saturation magnetization is due to the divalent ion present at the octahedral ' $\mathrm{B}$ ' sites. In the case of CFO, tetrahedral 'A' sites are occupied by $\mathrm{Fe}^{3+}$ ions and octahedral ' $\mathrm{B}$ ' sites are occupied by $\mathrm{Co}^{2+}$ and $\mathrm{Fe}^{3+}$ ions. The magnetic moments of ' $\mathrm{A}$ ' and ' $\mathrm{B}$ ' sites interact via antiferromagnetic interaction. Hence, magnetization due to $\mathrm{Fe}^{3+}$ ions in 'A' and ' $\mathrm{B}$ ' sites are cancelled and only the $\mathrm{Co}^{2+}$ ions contribute to the net magnetization in CFO system. As per literature, the theoretical value of net magnetization due to divalent $\mathrm{Co}^{2+}$ ions per formula unit of $\mathrm{CFO}$ is $3 \mu_{\mathrm{B}}$ and the measured magnetization of CFO nanoparticles is $3.7 \mu_{\mathrm{B}}$ [27]. These values are exactly similar to our calculated value of magnetization. The high value 653 Oe of coercive field is also observed for the CFO nanoparticles. These high values of saturation magnetization and coercivity may be due to the shape anisotropy because of the cuboid structure of CFO nanoparticles.

The hysteresis behaviour of 4,8 and $10 \mathrm{wt} \%$ of PVA$\mathrm{CFO}$ nanocomposite films at two different temperatures of 10 and $300 \mathrm{~K}$ are shown in figure $8 \mathrm{a}$ and $\mathrm{b}$, respectively. This hysteresis nature indicates the existence of ferromagnetic properties and the spontaneous magnetization of the PVA-CFO nanocomposites. It is observed that at low temperature, the values of saturation magnetization $\left(M_{\mathrm{S}}\right)$, remanent magnetization $\left(M_{\mathrm{R}}\right)$ and the coercive field $\left(H_{\mathrm{C}}\right)$ are higher and this is due to the reduction of thermal fluctuation of the magnetic dipoles. The values of $M_{\mathrm{S}}, M_{\mathrm{R}}$ and $H_{\mathrm{C}}$ for all the nanocomposites and CFO nanoparticles are summarized in table 3 . It is observed that the measured coercivity of pure CFO nanoparticles is low as compared to the 
Table 2. Estimated parameters $\tau, \beta, m, \sigma_{\mathrm{sp}}$ and $\sigma_{\mathrm{fc}}$ from the least-square fitting of the modified Cole-Cole equation of real and imaginary parts of the dielectric permittivity.

\begin{tabular}{lcccccc}
\hline Nanocomposites & $T(\mathrm{~K})$ & $\tau(\mathrm{s})$ & $\beta$ & $m$ & $\sigma_{\mathrm{sp}}\left(\mathrm{s} \mathrm{m}^{-1}\right)$ & $\sigma_{\mathrm{fc}}\left(\mathrm{s} \mathrm{m}^{-1}\right)$ \\
\hline S-0 & 363 & $6.35 \times 10^{-4}$ & 0.99 & 0.65 & $1.37 \times 10^{-7}$ & $2.02 \times 10^{-6}$ \\
& 373 & $1.76 \times 10^{-4}$ & 0.96 & 0.79 & $8.65 \times 10^{-7}$ & $5.55 \times 10^{-6}$ \\
& 383 & $7.67 \times 10^{-5}$ & 0.96 & 0.81 & $1.84 \times 10^{-6}$ & $1.06 \times 10^{-5}$ \\
& 393 & $4.27 \times 10^{-5}$ & 0.94 & 0.84 & $3.01 \times 10^{-6}$ & $1.56 \times 10^{-5}$ \\
& 403 & $3.45 \times 10^{-5}$ & 0.92 & 0.85 & $3.73 \times 10^{-6}$ & $2.69 \times 10^{-5}$ \\
S-4 & 363 & $8.99 \times 10^{-4}$ & 0.99 & 0.60 & $9.57 \times 10^{-8}$ & $1.15 \times 10^{-6}$ \\
& 373 & $3.68 \times 10^{-4}$ & 0.97 & 0.72 & $2.97 \times 10^{-7}$ & $3.12 \times 10^{-6}$ \\
& 383 & $1.89 \times 10^{-4}$ & 0.95 & 0.75 & $5.88 \times 10^{-7}$ & $6.47 \times 10^{-6}$ \\
& 393 & $9.55 \times 10^{-5}$ & 0.95 & 0.79 & $1.44 \times 10^{-6}$ & $8.65 \times 10^{-6}$ \\
S-8 & 403 & $4.45 \times 10^{-5}$ & 0.93 & 0.82 & $3.03 \times 10^{-6}$ & $1.55 \times 10^{-5}$ \\
& 363 & $9.41 \times 10^{-4}$ & 0.98 & 0.60 & $8.69 \times 10^{-8}$ & $1.87 \times 10^{-6}$ \\
& 373 & $2.53 \times 10^{-4}$ & 0.98 & 0.69 & $3.01 \times 10^{-7}$ & $4.45 \times 10^{-6}$ \\
& 383 & $1.45 \times 10^{-4}$ & 0.96 & 0.76 & $7.88 \times 10^{-7}$ & $6.93 \times 10^{-6}$ \\
& 393 & $9.05 \times 10^{-5}$ & 0.95 & 0.77 & $1.30 \times 10^{-6}$ & $1.01 \times 10^{-5}$ \\
S-10 & 403 & $3.62 \times 10^{-5}$ & 0.94 & 0.81 & $3.09 \times 10^{-6}$ & $2.63 \times 10^{-5}$ \\
& 363 & $3.52 \times 10^{-4}$ & 0.98 & 0.68 & $5.19 \times 10^{-7}$ & $3.33 \times 10^{-6}$ \\
& 373 & $2.73 \times 10^{-4}$ & 0.96 & 0.73 & $7.57 \times 10^{-7}$ & $5.87 \times 10^{-6}$ \\
& 383 & $1.73 \times 10^{-4}$ & 0.95 & 0.78 & $1.66 \times 10^{-6}$ & $1.02 \times 10^{-5}$ \\
& 393 & $1.15 \times 10^{-4}$ & 0.92 & 0.87 & $3.10 \times 10^{-6}$ & $1.35 \times 10^{-5}$ \\
& 403 & $4.69 \times 10^{-5}$ & 0.92 & 0.90 & $7.69 \times 10^{-6}$ & $3.61 \times 10^{-5}$ \\
\hline
\end{tabular}
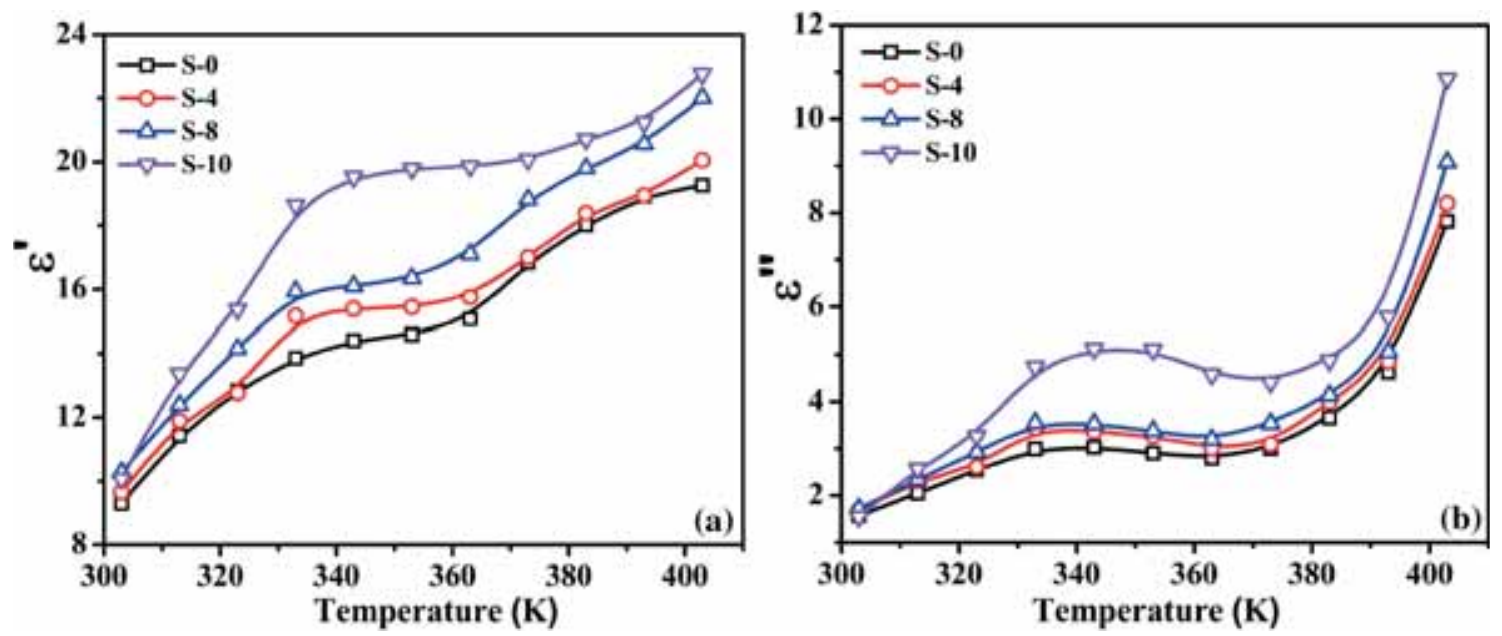

Figure 6. Variations of (a) real and (b) imaginary parts of dielectric permittivity with varying temperatures for S- 0 , S-4, S-8 and S-10 at $1 \mathrm{MHz}$ frequency.

coercivity of PVA-CFO nanocomposites. By increasing the $\mathrm{CFO}$ filler concentration in PVA-CFO nanocomposites, the coercivity value is increased. We expect that the enhancement of the coercivity in polymer phase is due to the intensive effect of magnetostrictive coupling. First, the magnetostrictive effect induces a distortion of the crystal lattices of CFO under an applied magnetic field, which in turn leads to local strains or stresses between the polymer and magnetic phases via intimate mechanical contact. When the material is subjected to an external magnetic field, the domain orientation and grain boundary movement occurred and due to this, the shape and size of the material will change. In this work, PVA matrix trapped the CFO nanoparticles and when the external magnetic field is applied, domain of CFO nanoparticles try to align in the direction of applied magnetic field and started the grain boundary movement, but the polymer matrix restricted 
the movement of grain boundary and at the interface between the polymer matrix and the CFO nanoparticles, local strain or stress is generated. The interfacial stress is inversely applied to the CFO phase which further leads to the change of domain magnetization as a result of an inverse magnetostrictive effect. This is consistent with the previous reports. Salunkhe et al [11]

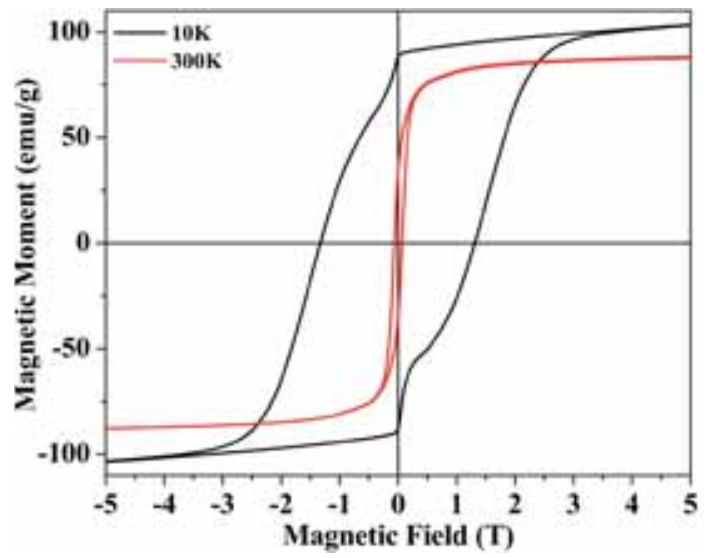

Figure 7. $\mathrm{M}-\mathrm{H}$ curves of $\mathrm{CFO}$ nanoparticles at temperatures of 10 and $300 \mathrm{~K}$.

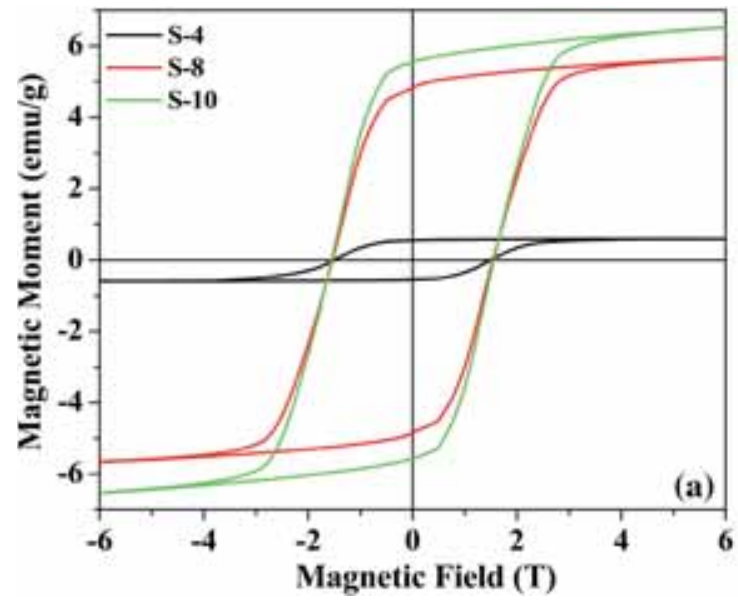

have also observed the increasing nature of coercivity i.e., 94 and 102 Oe for CFO nanoparticles and surface coating of PVA on CFO nanoparticles, respectively, at room temperature. Rashidi et al [28] got the coercivity value of 832 Oe for pure CFO nanoparticles, whereas for PVA/CoFe ${ }_{2} \mathrm{O}_{4}$ nanocomposites prepared via mechanical alloying method are varying from 506, 519, 540 Oe for PVA-CFO 8, 5 and $2 \mathrm{wt} \%$, respectively, with milling time $10 \mathrm{~h}$ and PVA-CFO $2 \mathrm{wt} \%$, the coercivity value is 507 Oe for $30 \mathrm{~h}$ milling time.

\section{Conclusions}

CFO nanoparticles and PVA-CFO nanocomposite films were synthesized successfully by wet chemical method. The samples were characterized by XRD and TEM, which confirm the formation of nanoparticles with cuboid shape. The variation in $\mathrm{AC}$ conductivity is measured as a function of frequency ranging from $100 \mathrm{~Hz}$ to $1 \mathrm{MHz}$ and temperature ranging from 303 to $403 \mathrm{~K}$. From the AC electrical transport properties, it was observed that the frequency exponent $S$ decreased according to increasing temperature and this is explained by $\mathrm{CBH}$ model. The corresponding maximum barrier height and relaxation time of the nanocomposites are calculated.

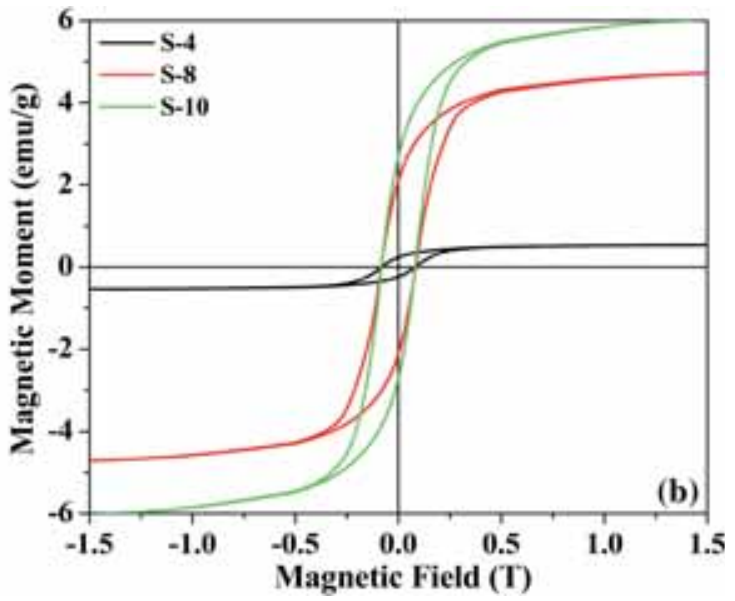

Figure 8. M-H curves of S-4, S-8, S-10 at varying temperatures of (a) 10 and (b) $300 \mathrm{~K}$.

Table 3. Estimated saturation magnetization $\left(M_{\mathrm{S}}\right)$, remnant magnetization $\left(M_{\mathrm{R}}\right)$ and coercive field $\left(H_{\mathrm{C}}\right)$ of S- - , S-4, S-8, S-10 and cobalt ferrite nanoparticles at temperatures 10 and $300 \mathrm{~K}$.

\begin{tabular}{|c|c|c|c|c|c|c|}
\hline \multirow[b]{2}{*}{ Samples } & \multicolumn{2}{|c|}{$M_{\mathrm{S}}\left(\mathrm{emu} \mathrm{g}^{-1}\right)$} & \multicolumn{2}{|c|}{$M_{\mathrm{R}}\left(\mathrm{emu} \mathrm{g}^{-1}\right)$} & \multicolumn{2}{|c|}{$H_{\mathrm{C}}$ (Tesla) } \\
\hline & $10 \mathrm{~K}$ & $300 \mathrm{~K}$ & $10 \mathrm{~K}$ & $300 \mathrm{~K}$ & $10 \mathrm{~K}$ & $300 \mathrm{~K}$ \\
\hline S-4 & 0.5869 & 0.4997 & 0.5518 & 0.2377 & 1.4994 & 0.0811 \\
\hline S-8 & 5.7111 & 4.8408 & 4.8347 & 2.1018 & 1.5388 & 0.0839 \\
\hline S-10 & 6.6075 & 6.3305 & 5.5560 & 2.6922 & 1.5553 & 0.0819 \\
\hline CFO nanoparticles & 106.32 & 89.16 & 87.95 & 34.38 & 1.3222 & 0.0653 \\
\hline
\end{tabular}


Frequency dependence real and imaginary parts of dielectric permittivity with different temperatures for PVACFO nanocomposite films were studied on the basis of the modified Cole-Cole model. The high value of $89 \mathrm{emu}^{-1}$ of saturation magnetization, 652 Oe coercivity are observed in CFO nanoparticles. The coercivity is increased in the case of PVA-CFO nanocomposites as compared to CFO nanoparticles, which are expected due to inverse magnetostrictive effect. The dielectric permittivity and magnetization of the nanocomposites are increased with the increase in CFO concentration, which enhances their application potentiality in miniaturization of the antenna system.

\section{Acknowledgements}

We would like to acknowledge the Centre of Excellence in Advanced Materials, TEQIP II, National Institute of Technology, Durgapur, for financial support and UGC-DAE Consortium for Scientific Research, Indore, for providing the magnetic measurement facility.

\section{References}

[1] Leslie-Pelecky D L and Rieke R D 1996 Chem. Mater. 81770

[2] Terris B D and Thomson T 2005 J. Phys. D: Appl. Phys. 38 199

[3] Chow E, Lin S Y, Johnson S G, Villeneuve P R, Joannopoulos J D, Wendt J R et al 2000 Nature 407983

[4] Zhu Y G, Li Z Q, Zhang D and Tanimoto T 2006 J. Appl. Polym. Sci. 99501

[5] Zhu Y G, Li Z Q, Gu J J, Zhang D and Tanimoto T 2006 J. Polym. Sci., Part B: Polym. Phys. 443157

[6] Khairy M and Gouda M E 2015 J. Adv. Res. 6555
[7] Covaliu C I, Jitaru I, Paraschiv G, Vasile E, Biris S S, Diamandescu L et al 2013 Powder Technol. 237415

[8] Qin R, Li F, Liu L and Jiang W 2009 J. Alloys Compd. 482508

[9] Prasanna G D, Jayanna H S and Prasad V 2011 J. Appl. Polym. Sci. 1202856

[10] Kayal S and Ramanujan R V 2010 Mater. Sci. Eng. C 30484

[11] Salunkhe A B, Khot V M, Thorat N D, Phadatare M R, Sathish C I, Dhawale D S et al 2013 Appl. Surf. Sci. 264598

[12] García-Cerda L A, Escareño-Castro M U and Salazar-Zertuche M 2007 J. Non-Cryst. Solids $\mathbf{3 5 3} 808$

[13] Vadivel M, Babu R R, Arivanandhan M, Ramamurthi K and Hayakawa Y 2015 RSC Adv. 527060

[14] Sinha S, Chatterjee S K, Ghosh J and Meikap A K 2015 J. Mater. Sci. 501632

[15] Cullity B D and Stock S R 2001 Elements of X-ray diffraction 3rd ed (England, UK: Pearson Education Limited)

[16] Mott N F and Davis E A 1979 Electronic processes in noncrystalline materials 2nd ed (New York: Oxford University Press)

[17] Elliott S R 1987 Adv. Phys. 36135

[18] Efros A L 1981 Philos. Mag. B 43829

[19] Jonscher A K 1977 Nature 267673

[20] Long A R 1982 Adv. Phys. 31553

[21] Koops C G 1951 Phys. Rev. 83121

[22] Hemalatha K S, Sriprakash G, Ambika Prasad M V N, Damle R and Rukmani K 2015 J. Appl. Phys. 118154103

[23] Sinha S, Chatterjee S K, Ghosh J and Meikap A K 2014 J. Phys. D: Appl. Phys. 47275301

[24] Chithra M, Anumol C N, Sahu B and Sahoo S C 2016 J. Magn. Magn. Mater. 4011

[25] Vázquez-Vázquez C, López-Quintela M A, Buján-Núñez M C and Rivas J 2011 J. Nanopart. Res. 131663

[26] Long N V, Yang Y, Teranishi T, Thi C M, Cao Y and Nogami M 2015 RSC Adv. 556560

[27] Cullity B D and Graham C D 2009 Introduction to magnetic materials 2nd ed (New Jersey, USA: IEEE Press, John Wiley \& Sons, Inc.)

[28] Rashidi S and Ataie A 2016 Mater. Res. Bull. 80321 